\title{
LES MARCHANDS FLAMANDS ET LE ROI D'ANGLETERRE
}

\section{(1 $154-1216)$}

Bien que nous n'ayons que fort peu de textes qui nous parlent du commerce anglo-flamand aux $X^{e}, X^{e}$ et au début du XIIe siècles, nous pouvons conjecturer qu'il fut toujours assez important.

Dès la fin du $\mathrm{X}^{\mathrm{e}}$ siècle, les Flamands sont cités parmi les marchands qui visitent l'Angleterre (1); on les y retrouve régulièrement avant 1066 (2) : ils y portent des marchandises de toute espèce et en ramènent des objets d'orfèvrerie et des broderies (3).

La conquête de Guillaume de Normandie intensifia sans aucun doute les rapports de la Flandre et de l'Angleterre tant au point de vue économique que politique. Fn 1114, on nous parle encore de marchands " de regno Balduini venientes "(4). En 1.127, les Flamands sont si nombreux à Londres, la circulation entre l'Angleterre et. la Flandre est si intense que deux jours après le meurtre de Charles le Bon, la nouvelle en est connue dans la capitale anglaise (5). Vers la même époque, les marchands flamands se rendent déjà par groupes en Angleterre pour. y acheter de grandes quantités de laine (6).

Il semble certain que la formidable anarchie du

(1) Hansisches Urkundenbuch 55, t. I, p. 2, n. 2.

(2) Notamment en 1050 : Miracula Sancti Trudonuis, Mon. Germ. Hist. t. XV, p. 827.

(3) Cf. Green, History of the Finglish people, t. I, p. 153.

(4) Hans. Urk., t. I, p. 3, n. 5.

(5) Galbert de Bruges, éd. Pirenne, p. 22 et n. 2.

(6) IIermannide miraculis $\mathrm{S}$. marir laudunensis. Migne Patrologie t. CI.VI, pp. 975-77. 
règne d'Etienne de Blois (1.135-1154) (1) provoqua la ruine momentanée du commerce anglo-flamand.

Aurait-il pu en être autrement? Jamais peut-être l'insécurité des routes ne fut plus grande en Angleterre. De tous côtés on construit des châteaux forts qui deviennent de véritables repaires de brigands. Des troupes armées en battent sans cesse les environs : partisans et adversaires du roi harcèlent les voyageurs et les habitants, leur arrachent tout ce qu'ils possèdent et se font un jeu de les soumettre aux tortures les moins imaginables (2). Jamais la force brutale ne fut à pareille fête. Les mercenaires du roi, loin de maintenir l'ordre, rivalisent de cruauté avec les organisateurs de l'anarchie.

Quels marchands flamands auraient eu la folie de s'aventurer dans un pays où le pillage et le meurtre étaient devenus lois? C.e n'étaient pas les mercenaires flamands, les plus cruels de tous, qui les auraient préservés des dangers. Dirigés par Guillaume d'Ypres, provoquant dans toute l'Angleterre une terreur sans bornes, ils ne connaissaient ni amis, ni compatriotes quand il s'agissait de rapines! (3).

(1) L'histoire d'Etienne de Blois est cncore à faire. Mais M. Round, dans son excellent livre Geoffrey de Mandeville. A study of anarchy, London, 1882. in-8 $8^{\circ}$ a donné un tableau saisissant et en tous points remarquable de la longue période de luttes intestines qui précède l'avènement de Henri 11.

(2) Anglo-saxon chronicle éd. et trad. 13. Thorpe. Roll Series (12. S.) II, pp. $230-31$.

(3) Sur les merecnaires flamands de l'arméc d'Wtienno et sur leur chef (Guillaume d'Ypres (le prétendant au trône comtal de Flandre en 1127), voir notamment Gervais de Canterbury, Chronic. ed. Stubbs R. S., 1, pp. 95, 105, 161. -Ordéric Vital, Hist. Eccles.. ed. Le Prévost, t. V. pp. 81, 83, 84. Aclred de Ricvaulx. Relatio de Standardo ed. Howlett. Chronicles of the reign of Stephen, II enri II and Richard Ier, 12. S., t. III, p. 182. - Raoul de Diceto éd. Stubbs (IR. S.), I, p. 297. - Robert de Torigny. Chronica éd. Délisle t. 1, p. 291. Les mercenaires flamands "famelicorim more luporum fecun ditalem terrae Anglicanae ad nichilum redigere studuerunt, "Gorvais de. Canterbury, op. cit., l, p. III. Les chemins étaient si peu sûrs on 11/ « « ut nec etiam episcopi nec monachi devilla in villam tuto possent progredi". Guillaume de Malmesbury Historiae novellae, éd. Stubbs (R.S.), II,p.561,, RôssLer-Kaiserin Malhilde, p. 189 , dit que ce fut une des grandes fautes d'Etienne de Blois de s'appuyer sur les mercenaires flamands. Je pense au contraire que sans cux il lui aurait été impossible de résister à ses adversaires pendant près de vingt ans. 
LES MARCHANDS FI.AMANDSE'T LE ROI D' ANGLETERRE 305

Aussi, pendant près de vingt ans, ne trouvons-nous aucune trace d'un commerce flamand en Angleterre. Alors que les chroniqueurs étaient assez loquaces, jusqu'en 1135 au sujet des " mercatores Flandriae" et des "Flandrenses ", ils n'en parlent plus après cette date si ce n'est pour comparer leur attitude toute pacifique sous Henri Ier, aux n.œurs barbares des soldats flamands d'Etienne "rapto vipere assueti "(1). Il y eut bien sans doute quelques colporteurs flamands, semblables à ceux qui, en 1215, trâ̂nèrent derrière les troupes de Jean sans Terre (2), pour suivre les mercenaires d'Etienne et organiser un petit trafic qui ne manque pas d'intérêt. Mais comme ils n'avaient probablement pas de rapports avec les habitants, les contemporains ne nous en ont pas parlé : on les aura considérés comme faisant partie des bandes armées qu'ils accompagnaient.

La réconciliation d'Etienne. de Blois et de son rival (1153) et l'avènement de celui-ci (1154) furent les préludes d'une nouvelle ère de paix et de sécurité. En quelques mois, Henri II réussit à soumettre tous les nobles; il ordonne la destruction des châteaux forts construits sans autorisation royale pendant la période des troubles et supprime ainsi une des causes les plus profondes de l'insécurité des routes (3); enfin, il renvoie tous les mercenaires flamands, conduits par Guillaume d'Ypres qui pleurait (4) :

(1) Guillaume de Malmesbury, op. cet. t. II, p. 561.

(2) Il y eut sous Jean sans Ter re une véritable avalanche de mercenaires flamands - chevaliers ou non - en Angleterre. Comme on peut le voir par les Rotuli litterarum patentium (éd. Hardy, Londres, 1835) et les Rotuli litterarum clausarum (éd. Hardy, Londres, 1833). Il est impossible d'entrer ici dans des détails. Pour une idée générale, cf. Stuввs, Histoire Constitutionnelle de l'Angteterre, trad. Petit-Du tainiris, t. I, pp. 529, 703. En1215-16, des marchands flamands vendaient beaucoup aux mercenaires et passaient de l'armée du roi Jean à celle du prince Louis. Rot. litt. pat., pp. 158 b. et 176 b. (bis).

(3) D'après Davis, England under the Normans and Angevins, p. 179, 1115 châteaux auraient été détruits en peu de temps. Cf. Sturrs-PeritDutaillis, op. cit., p. 548 .

(4) "Willelmus de Ypra, violentius incubator Cantiae cum lacrymis emigrav it". Willelmus filius Stephani. Vita S. Thomae, éd. Robertson Matérials for the history of Th. Becket (R. S.), t. III, p. 19. Henri II fut d'ailleurs obligé. de se servir lui aussi des mercenaires, et cela dès 1160. Annales cambriae, éd. Williams (R. S.), p. 50. Cf. Pipe Roll 1173 f74, éd. Pipe Roll Society, t. XXI, pp. 52 et 135. 
ces pillards quittèrent l'Angleterre en si grande hâte qu'on les aurait crus en fuite (1).

Il n'en fallut pas plus pour rendre confiance aux bourgeois et aux paysans : les terres sont de nouveau cultivées, les villes regagnent leur ancienne prospérité, et dès 1155 , Raoul de Diceto peut célébrer la renaissance commerciale de son pays (2).

Les marchands flamands reprirent très vite le chemin de l'Angleterre; déjà en 1154, 'Thierry d'Alsace avait eu une entrevue amicale avec Henri Il (3); le traité de Douvres (19 mars 1163) vint encore resserrer les liens qui unissaient le eomte au roi (4) : la situation était excellente pour l'expansion économique flamande.

En 1155, Guillaume Cade, bourgeois de Saint-Omer, jouit de la faveur du roi (5). Bientôt les hommes de SaintOmer (1155-58) reçoivent l'autorisation de faire du commerce à Londres et dans toute l'Angleterre (6). Dès avant 1166 , près de vingt flamands sont signalés comme ayant empruntè de l'argent dans la capitale anglaise (7). En 1173, le commerce anglo-flamand est devenu assez important pour que Henri II croie utile de faire saisir les biens des marchands flamands (8); avec Richard Ier

(1) Guillaume de Newburgh, Historia rerım anglicarum, éd. Ifowlett, op. cit., I, p. 101.

(2) Raoul de Diceto, éd. Stubis, I, p. 297. - Davis, op. cil., p. 201.

(3) Lors de son couronnement. Robert de Torigny, op. cit., t. I, p. 290. D'autres entre vues eurent lieu en 1156 et 1157 , ibidem pp. 297 el 307 . N'oublions pas queThierry d'Alsace avait épousé Sibylle d'Anjou, tante de Henrill.

('t) Delisis, Recueil des actes de Henri 1I, t. I, pp. 375-6. Ce traité qui a une importance capitale renouvelait l'alliance anglo-flamande du débul du XIJc siècle. Il fut repris en 1180. Gesta Henrici II, éd. Sterns (R. S.), I, Pl. 2:6-7.

(5) Voir note 13 et p. 15.

(6) Deirisle, Recueil, t. I, p. 173, no LXXI.

(7) Debita Willelmi Cade, éd. Jenkrson, E. II. R., I. XXVIII (1913), pp. 22/1-27. Guillaume CaDre habitait probablement. Londres at certainement l'Angleterre.

(8) Pipe Rolls, 1172-73, Pipe Rolls, Soc. t. XIX, pp. 13, 29, 50, 130, 171, 196. Pipe Rools 1173-71 ; ibidem, t. XX I, p. 50. 
LES MARCHAN DS FIAMANDS ET LE ROI D' ANGLETERRE $30 \overline{7}$

et Jean sans Terre les traces en sont de plus en plus nombreuses (1).

Mais si les relations commerciales de la Flandre et de l'Angleterre ont repris après 1154 avec plus d'intensité que sous Henri Ier, il s'en faut de beaucoup qu'elles se sòient rétablies dans les mêmes conditions.

D'abord les villes tant anglaises que flamandes se sont développées. Cette situation nouvelle présente, pour les marchands flamands, un immense avantage : ils ne sont plus désormais des individus isolés ou des groupes d'hommes vaguement connus sous le nom de "mercatores " ou de "Flandrenses", obligés de pourvoir euxmêmes à leur sécurité et ne pouvant compter en pays étranger que sur de maigres appuis. "Ils représentent maintenant leurs villes qui sont connues en Angleterre comme étant des plus riches et des plus puissantes. Ils sont bourgeois de Bruges, de Gand, d'Ypres ou de SaintOmer et c'est en cette qualité qu'ils sont accueillis par les bourgeois anglais (2). De plus, ils trouvent en Angleterre des organismes parfaitement semblables à ceux de leur comté et cette similitude d'institutions leur rend de grands services.

Mais il y a une ombre à ce tableau : le développement des villes anglaises commence à être un obstacle au commerce étranger. A mesure que les bourgeois anglais s'enrichissent et que leurs privilèges deviennent plus nombreux, ils cherchent à étendre leurs affaires et à évincer les concurrents étrangers du trafic intérieur. Ils ne demandent pas mieux que de les recevoir puisqu'ils exportent les produits bruts du pays et qu'ils y appor-

(1) Pour un résumé - d'ailleurs incomplet - ef. Varenbergh, Histoire des relations diplomatiques entre le comté de Flandre et l'Angleterre au Moyen Age (1874), p.91 sqq.

(2) A partir du dernier quart du XIle siècle, on commence, dans les sources anglaises à les appeler non plus "Flandrenses ", mais "burgenses" ou "mercatores" de telle ou de telle ville. Sous Jeansans Terre c'est toujours ainsi qu'on leś cite à quelques rares exceptions près. 
tent les fins draps de Flandre et les vins de Gascogne. Mais ils veulent coûte que conte garder le monopole de la vente au détail et forcer chacun à leur acheter en gros les cuirs et laines d'exportation. Entre marchands étrangers et indigènes, c'est une lutte sourde; on s'en rend compte dès le règne de Henri ler _..-privilège de Newcastleon-Tyne (1) - mais elle s'amplifie surtout sous Henri II : grâce au soutien du roi, les villes anglaises l'emportent et une série de privilèges urbains limite l'activité des marchands d'outre-mer (2).

Ce n'est d'ailleurs pas l'attitude des villes anglaises qui gêne le plus les Flamands : le protectionnisme leur est familier; de plus, il est une conséquence de la puissance des boroughs grâce à laquelle le commerce a gagné en stabilité et en étendue. Ce qui vraiment les paralyse, c'est l'intervention continuelle de l'administration royale dans leurs affaires.

Jusqu'au milieu du XIfe siècle, si un mercator payait les taxes qu'on exigeait de lui, il pouvait circuler librement (3). Il avait, il est vrai à pourvoir à sa propre sécurité, - et ce n'était pas toujours chose aiséc ; mais il allait où il voulait, vendait ses marchandises comme il voulait et pouvait compter la plupart du temps sur la protection royale.

Cette situation changea à la fin du XIIe siècle par l'intrusion de la politique dans le domaine économique. Henri II déjà avait compris que le commerce étranger pouvait être pour lui non seulement une immense source

(1) BAithand, British Borough Charters, p. 211.

(2) Exemples dans Balland, op cit., pp. 201 à 216. Cf. Ashley, Histoire et doctrine économiques de l'Angleterre, trad. Pondois, t. I, pp. 141-142. Lipson, Economic history, t. T, p. $4_{4} 48$. Jean sans Terre continua la politique de Henri IJ. Ce fut lui qui en 1200 avertit le marchand étranger qu'il aurait droit ì la même protection que celle dont jouiraient les marchands anglais dans son pays. Rot. Char tarum, p. 606. Il ne cessa pas d'appliquer cette règle. Én 1215 un paragraphe de Magna Carta fut consacré au commerce. Mac Kechnie, Magna Carta, $2^{\mathrm{e}}$ éd. 1914, pp. 463-473 en a donné un commentaire.

(3) Sur les taxes en Angleterre au XI $I^{e}$ siècle, cf. Gras, The eswly English custom system (Harvard Economic Studies, t. XVIII), 1918, p. 31 sqq. 
de revenus, mais aussi un moyen d'exercer une pression politique sur des états voisins : en 1173, il saisit les biens des marchands flamands qui se trouvaient en Angleterre uniquement, semble-t-il, pour forcer Philippe d'Alsace à abandonner ses fils révoltés (1).

Mais ce fut Jean sans Terre qui lia la vie économique de l'Angleterre à ses combinaisons politiques : pour affaiblir la résistance de ses ennemis, il défend à leurs bourgeois d'entrer dans l'île et d'y faire du commerce (2), et pour protéger les villes anglaises en cas de conflit, il décide que les marchands étrangers seront traités en Angleterre de la même façon que les marchands anglais auront été traités chez eux (3). Ainsi -vit le jour la politique de pression économique qui subsistera à travers tout le Moyen Age et qui aura tant de succès sous Edouard III.

Désormais, plus de sécurité pour les Flamands qui trafiquent dans l'île : il suffit que le comte de Flandre semble se rapprocher du roi de France pour qu'aussitôt Jean sans Terre interdise aux bourgeois flamands de venir en Angleterre ou s'empare des biens de ceux qui y voyagent; c'est assez qu'un Anglais ait été mis en état d'arrestation en Flandre pour que Jean sans Terre exerce des représailles (4).

Et le roi ne s'en tient pas là. Il établit toute une réglementation commercialc. Il augmente les taxes, interdit l'exportation de certains produits, sauf licence, et fait surveiller étroitement les ports de mer : l'Assise de Winchester du 4 juin 1204 marque le point culminant de ce régime (5) : désormais, toutes les marchandises impor-

(1) Voir note 15.

(2) Exemples caractéristiques. Rout litı. clans, I, p. 145 et surtout ibidem, 1, 209 b et 211 .

(3) Rot. chartorum, p. 60 b.

(4) Notamment en 1208 ; voir plus loin note 49.

(5) Rot. litt. pal., p. 42. Gras, op. cit.; p. $48 \mathrm{sqq}$. a réccmment étudié cette Assise du point de vue financier et l'a rééditée, p. 218 sqq. Il pense (p. 50) que l'impôt du quinzième fut supprimé entre 1207 et 1210. On peut 
tées ou exportées seront soumises à un nouvel impôt en nature dit du quinzième (quindena); dans chaque port, six bourgeois ou plus, 1 chevalier et 1 clerc (ballivi quindenae) seront élus pour recevoir et garder cet impôt ; ils seront responsables vis-à-vis de trois fonctionnaires supérieurs "capitales custodes portuum et quindenae "(1); rien ne pourra être chargé ou déchargé sans l'autorisation des baillis; personne ne pourra acheter la laine des abbayes, ni exporter le blé, le vin, le jambon, le beurre, le fromage et quelques autres produits sans la permission du roi ou des capitales custodes.

Pour mieux permettre la stricte exécution de son Assise, Jean sans Terre prend des mesures rigoureuses de surveillance. C'est chose assez aisée puisque l'Angleterre est une île. 11 suffit de lire le récit des événements qui ont suivi le meurtre de Becket (1170) pour voir que déjà sous Henri II la côte était si bien gardée qu’il était vraiment difficile d'y débarquer clandestinement (2). Sous Jean sans 'Terre, elle l'est mieux que jamais (3). Après la perte de la Normandie, la flotte est réorganisée et les baillis des ports sont harcelés de recommandations. Les marins sont sans cesse appelés au service du roi et obligés de patrouiller pour son compte dans la mer du Nord (4) : il est quasi impossible pour un

cn douter : en tout cas l'exportation de la laine, du blé etc., contimua à être surveillée et les ballivi quindenae subsistèrent : c'est là ce qui importe ici. - Il n'y a aucune raison de dater l'Assise du ', juin 1203. Elle vient en effet en tête du rôle de la sixième annéc du roi Jean, année qui débute lo 3 juin 1204. Il est vrai que l'acte est daté "anno quinto ", mais cette erreur s'explique par l'inattention du scribe. Ajoutons qu'avant 1204, on ne trouve aucune trace de la quindena. Par contre, on en parle dès le 6 juin 1204. Rot. litt. pat., p. 43 .

(1) Deux d'entre eux, Renaud de Cornhili et Gulliaume de Wrotham claient des personnages importants. Pour le premicr. voir Diclionnary of national biography, t. IV, p. 1154 ; pour le second, ibidem, t. XXI, p. 1081.

(2) Gervais de Canterbury, op. cil., I, p. 234.

(3) Le roi fit établir des postes de surveillance jusque dans les îles anglonormandes " ad gravandum inimicos nostros". Rot. litt. pat.,p. 84.

(4) Ises marins se plaignirent plus tard que le roi "les ot mis en servage".

Histoire de Guillaume le Maréchal, ed. Meyer, iI, p. 258, vors 1723.

Ainsi le 17 mars 1208, Jean sans 'Terre ordonne aux baillis des ports de 
marchand d'entrer dans l'île sans sauf-conduit, et s'il y parvient, les baillis du quinzième l'empêchent de faire du commerce.

Toutes les mesures prises par Jean sans Terre affectent d'autant plus les marchands flamands que le commerce en Angleterre devient de plus en plus fructueux. Le blé que les Anglais vendent par immenses quantités, le vin que les bateaux flamands vont chercher à La Rochelle pour le revendre avec un considérable bénéfice soit en Angleterre, soit en Flandre; les cuirs anglais, si nombreux sur le marché : tout cela et. bien d'autres produits attire les Flamands (1).

Mais ce qui leur importe surtout c'est la laine anglaise supérieure à celle de France et d'Italie et dont ils ont besoin pour leur industrie drapière déjà si développée (2). Dès 1127, les marchands flamands très nombreux à Londres $y$ achètent de la laine pour de fortes sommes (3): depuis cette date, ce commerce n'a fait que s'intensifier. Au milieu du règne d'Henri Il, les transactions de l'espèce deviennent de plus en plus nombreuses (4), et en 1173 , les Flamands sont si bien connus comme acheteurs ordinaires de la laine, que Jordan Fantôme insinue que les Flamands venus en Angleterre pour aider les fils révoltés d'Henri II

choisir " de melioribus ot validioribus hominibus portuum vestrorum et qui bene sint armati ad eskippandas galias nostras ". Rot. litt. pat., p. 80. Sur la marine anglaise, cf. le livre très insuffisant de Nxcolas, History of the royal navy (1847), t. I, p. 144 sqq. Pour Jean sans Terre, on peut consulter un interessant article de $\mathbf{F}$. W. Brooks, William de Wrotham and the Office of keeper of the king's porls and galleys. E. H. R., 1925, t. XL, pp. 570-80.

(1) En 1200, "Dou royaume d'Angleterre viennent lainnes, cuirs, plons estains, charbon de roche, fromaige". Cartulaire de l'Estaple de Bruges, éd. Gilliodts, t. I, p. 19.

(2) Il suffira de renvoyer ici à G. Espinas et H. Pirenne; Recueil de documents relatifs à l'histoire de l'industrie drapière en Flandrs. Bruxelles, 1906-24, 4 vol. in-4o.

(3) Galbert de Bruges, Histoire du meurtre de Charles le Bon, éd. Pirenne, p. 22. - Hermanni de miraculis sanctae Mariae Laudunensis, Migne, Patrologie, CLVI, pp. 975-77.

(4) Comme le prouvent les saisies de laine achetéc par les flamands Pour les textes, voir note 8, p. 306 . 
n'y sont allés que pour voler la "leine d'Engleterre"; il va même jusqu'à prétendre que les troupes levées en Flandre par le comte de Leicester étaient composées de tisserands sans ouvrage par suite du manque de matière première! ( 1 .

D'ailleurs, la production de la laine -- "lana pretiosissima" comme dit Henri de Huntingdon vers 1155 (2) - s'est répandue d'une façon étonnante. Alors que dans le Domesday-book on trouve à peine quelques allusions à des moutons (3), il semble qu'à la fin du $\mathrm{XII}{ }^{e}$ siècle, on en rencontrait partout où l'élevage était possible. M. Whitwell a montré que les Cisterciens en possédaient de grands troupeaux (4). On peut ajouter que dans les autres domaines ecclésiastiques et dans les domaines laïques on s'occupait tout autant d'élevage que dans ceux des Cisterciens. Nous en avons une preuve décisive dans les "Rotuli de dominabus et pueris et puellis de $X I I$ comitatibus ", relevé de terres appartenant aux veuves et aux enfants mineurs et dressé dans le but de fixer les droits du roi (5). On y voit que - à 9 exceptions près - tous les domaines qui ne sont pas trop petits pour permettre l'élevage du bétail possèdent des

(1) Voici le texte. Le chroniqueur fait parler un flamand: "Nus n'eimes pas en cest païs venuz pur sujorner, Mès pur lei rei destruire Henri le vielz guerrier. E pur aver sa leine dunt avumt desirier" ".

Et le chroniqueur ajoute :

"Seignurs co est la vérité : li plus furent tclier Ne saveient porter arnies a lei de chevalier".

Jordan Fantosme, Chronique de la guerre entre les Anglais et les licossais éd. Howlett, chron. etc. III, p. 288 vers 999 sqq. Cf. aussi pp. 290-292. Notons, que Jordan a écrit avant 1183.

(2) Henri de Huntingdon, Historia Anglorum, éd. Arnold, pp. 5-6.

(3) Domesday book, éd. Farley-Ellis, I, p. 354 h. : " Habet Gislebertus gregem ovium ". Cf. Whitwell, English monasieries and the wool trade in the XIII century. Viertelj. für Soz. und Writ. Gesch., II, pp. 13-14 (1204).

(1) Ibidem, p. 7 sqq.

(5) Ed. J.-H. Round, Pipe Rolls Society, t. XXXV, London 1913, in-8. Cf. introduction p. XXV. 
moutons : dans 96 domaines, il n'y en a pas moins de 15.000 (1).

Une grande partie de toute cette laine est vendue : les nobles (2) comme les ecclésiastiques (3) se procurent ainsi de l'argent. Les troupeaux des Cisterciens leur donnent de très beaux revenus qui servent plusieurs fois de prétexte à Richard ler pour extorquer de l'argent (4). Les plus pauvres essaient d'élever quelques moutons comme cette femme qui, vers 1172, destine l'argent que lui rapportera la vente de la laine de ses brebiss à un pèlerinage au tombeau de Saint Thomas à Canterbury (5).

Bref, la laine est déjà une des grandes richesses de l'Angleterre : c'est elle surtout qui attire les marchands flamands. Ils l'exportent par énormes quantités (6), et elle est si précieuse pour eux que lorsque le roi est dans l'impossibilité de leur rendre l'argent qu'ils lui ont prêté, il fait, à leur demande, une saisie de laine pour les indemniser (7).

Ainsi d'une part le commerce flamand en Angleterre devient de jour en jour plus intense; d'autre part le protectionnisme des villes, la règlementation et la poli-

(1) Il est vrai que souvent par suite de la n égligence de ceux qui avaicnt la garde des terres, le nombre des moutons a beaucoup diminué : il est tombé de 15.488 à 5.541. Voici un exemple : "Quando Adamus de Cardville recepit custodiam terre sue in Cattorpe, invenit ibi XXVIII boves quorum unus morturus fuit et DC oves de quibus $C C$ et $C X$ fuerunt vendite $X V \mathrm{~m}$. et $X I$ s et VIII $d$.; de residuis mortue fuerunt CC et quater XX quarum pelles vendite fuerunt III l. et XII s. Postea recepit Hugc de morewich custodiam de Cattorpe et invenit ibi... et $\mathrm{L}$ oves. "Rotuli de dominabus, p. 9 ".

(2) Cf. notamment Pipe Roll, 1183-84; Pipe Roll, Soc., t. XXXIII, p. 21, $110,155$.

(3) Un domaine ceclésiastique rapporte en 1181-2: 126 l. "de lana vendita". Pipe Roll. 1181-2; Pipe Roll, Soc. t. XXXI, p. 62. Cf. Rot. litt. pat. I, p. 81. Nombreux autres cas.

(4) Guillaumc de Newburgi, op. cil. Howlett Chron., elc. II, pP. 416-17; hoger de Hoveden, chronicon, éd. StubBs, R. S. II, p. 242. Cf. Wintwer., op. ri!., p. 2 sqq.

(5) "lanam ovicularam in riaticum destinavi". Guillaume DE CANTElBURy. Miracula S. Thomae, éd. Robertson Materials, I, p. 154.

(6) Rot. litu. claus., I, p. 90 : Simon Saphir exporte 80 sacs à la fois.

(7) Voir plus loin. 
tique économique royales rendent l'exercice de ce commerce de plus en plus difficile. Pour que les bourgeois de Flandre puissent continuer et étendre leur activité commerciale, il n'est qu'un moyen : il leur faut à tout prix obtenir la protection royale. Sans doute, cet appui ne brisera pas, pour eux, le monopole du commerce intérieur que les boroughs se sont acquis après tant d'efforts; mais le roi leur permettra d'aller en Angleterre et les prémunira contre les abus des villes et des fonctionnaires royaux ; il leur donnera des sauf-conduits et des licences d'exportation; qui sait, il les exemptera peut-être de l'impôt du quinzième (1).

Pour obtenir la faveur royale grâce à laquelle ils pourront continuer leur trafic en Angleterre, les marchands flamands sont prêts à toutes les concessions et à toutes les soumissions.

Il faut, avant tout, bien disposer le roi d'Angleterre pour la Flandre en général, et dans ce but, amener te comte à une politique favorable à l'Angleterre. Les bourgeois des grandes villes s'y efforcent. En 1195, ils semblent avoir contribué à l'alliance de Baudouin IX et de Richard Ier, alliance renouvelée en 1199 avec Jean sans 'Terre (2). Mais, c'est en 1208 que leur action politique apparaît pour la première fois décisive et d'une manière lumineuse : alors que le régent Philippe de Namur est aux ordres du roi de France, ils ne craignent pas de se déclarer les alliés de Jean sans Terre (3). Il faut dire que pour les y amener, celui-ci avait fait saisir par trois fois leurs marchandises en Angleterre! (4). Dès ce moment, ils ne cesseront plus de manifester leur sympathie pour le

(1) Comme Walter Spronck en 1204, Rot. litl.pat., p. lit.

(2) liymer. Fuedera, éd. La Haye, 1745, t. 1, p. 30. Cuntellient, Philipp Il August, t. III, p. 154 et p. 223 , no $261 ;$ p. 234 no 6 et p. 235, no 7.

(3) Champoltion-Figenc, Lettres de rois, etc. I, pp. 24-25 no 16, Cf. Pabst, Die aussere politik der grafschaft Flandern unter Ferrand uon Poriugal. B. (.). R. H., t. LXXX (1911), p. 82 .

(4) L.e 18 février (Rot. litt. claus. 1, p. 102 b), le 26 mai (Rot. litt. pat.. p. 83 b) et avant le 26 juillet (Rol. lill. pat., p. 85 b). 
roi Jean. En 1213, c'est leur influence tout autant que la rancune personnelle de Ferrand contre Philippe-Auguste et l'insistance des nobles flamands achetés par Jean sans Terre, qui jette le comte dans une nouvelle alliance anglaise (1). Et si, après Bouvines, leurs conseils ne sont que rarement suivis pendant près d'un demisiècle, c'est que la puissance du roi de France ne permetpas au comte de Flandre de les écouter.

Les villes sont d'autant plus disposées à intervenir en faveur du roi d'Angleterre, que leur action donne d'excellents résultats. Chaque fois qu'elles prouvent leur attachement au roi Jean, celui-ci les remercie en leur donnant ou en renouvelant une licence générale de commerce : ce fut le cas en 1208 et en 1213 (2).

Mais cela ne suffit pas pour trafiquer en toute liberté. Il faut encore se procurer, on l'a vu, des licences spéciales pour pouvoir effectuer certaines exportations. Ces licences sont individuelles et valables pour une fois seulernent : pour les obtenir, chaque marchand essaie d'entrer dans les bonnes grâces du roi. Il n'est rien qu'il ne fasse pour lui plaire, tous rivalisent de zèle à le servir. Les uns lui prêtent de l'argent (3) ; d'autres lui fournissent des chevaux (4), Simon Saphir sert d'agent de liaison entre le roi et ses partisans (5), un autre se fait' son ambassadeur auprès de son neveu Othon (6) ; Guillaume d'Ardenbourg et son frère transportent des envoyés d'Othon en Angleterre (7). Certains se distinguent particulièrement : tels Hugues Oysel, d'Ypres, Martin Campion, d'Arras, Simon Saphir et

(1) Rot. chartarum, I', p. 197.

(2) 13 septembre 1208 (Roll. chart, I', p. 132) et 17 avril 1213 (Kol. lill. pal., p. 98).

(3) P. ex. en 1199, Hugue Oysel prête 1700 livres au roi. Rot. Charl., 1', p. $11 \mathrm{~b}$.

(4) Rot. lill. pat., p. 77.

(5) Rot. litt. claus., J, p. 141.

(6) Rymer. Fodera, I1, p. 53.

(7) Rot. lilt. pat. p. 87 a. 
Walter Spronck, de Gand (1). Un des plus intéressants est Florent le Riche, de Saint-Omer. Voici, a son sujet, quelques notes biographiques.

Pour bien comprendre l'activité de Florent, de SaintOmer, il convient d'étudier brièvement les rapports de son milieu avec l'Angleterre

Saint-Omer avait, on le sait, des relations très étroites avec l'île d'outre-Manche. Admirablement située (2), elle semble avoir mis à profit la conquête de Guillaume le Conquérant pour étendre ses entreprises commerciales. En 1097 déjà les Anglais connaissaient bien le chemin de la ville (3); dès 1127, ses bourgeois faisaient promettre à Guillaume Cliton de les faire exempter de tout tonlieu s'il parvenait à se réconcilier avec Henri Ier (4). L'anarchie du règne d'Etienne fut désastreuse pour Saint-Omer comme pour les autres villes flamandes : mais dès l'avènement de Henri II, une belle compensation lui fut réservée qui devait donner à son commerce un grand essor. En effet, entre 1155 et 1158, Henri II lui accorda un privilège d'une grande importance : il donnait à ses bourgeois le droit d'avoir des "hospicia" à Londres; il leur permettait d'exercer leur commerce en toute liberté en Angleterre et les exemptait du lestage (5).

Il est impossible de savoir si Saint-Omer seule bénéficia à cette date d'une concession royale. Mais nous pouvons nous demander si la faveur qu'elle obtint ne fut pas due à l'influence d'un de ses bourgeois. En effet,

(1) Cf. Varcnbergh. Hist. des relations diplomatiques entre le comle de Flandre et l'Angleterre au Moyen Age (1874), p. 91 sqq.

(2) Cf. Giry. Histoire de Saint-Omer, p. $27 t_{\text {f }}$ et 276.

(3) C'est là que se réfugia en effet Anselme : Fadmer. Hist. novorum, éd. Rule R. S. p. 89 , ibidem Vita Anselmi. R. S. p. 387.

(4) Giry. Hist. de Saint-Omer, p. 372. P. J., no III.

(5) " et dissolvant sine licencia trussellos suos et cant ad nundinas et mercata et ferant et ducant res suas ad vendendum et emendum per tolam Angliam in mea firma pace et sint quieti per totam Angliam undecumque venerint de lestagio". Denisle, Recueil des actes de Henri II, t. I, p. 173, no 71. Cf. Giry, op. cit., p. 381, P. J., no VIII. 
vers cette époque, vivait à Londres Guillaume Cade, de Saint-Omer, qui rendait d'inappréciables services au roi (1). Grand usurier, il prêtait de l'argent à des dizaines de nobles anglais et surtout à Henri II lui-même ; mais il resta toujours fidèle à son pays d'origine où ses fils se retirèrent après sa mort (2). Ne fut-ce pas lui qui poussa le roi à gratifier Saint-Omer du privilège de 1155-58 ? C'est de la même façon que nous voyons Simon Saphir, cinquante ans plus tard, servir d'intermédiaire entre Jean sans Terre et les Gantois (3).

Quoiqu'il en soit, Saint-Omer profita de la faveur qu'elle venait d'obtenir pour développer son commerce d'outre-mer, et en 1165, nous voyons six bourgeois de Saint-Omer emprunter de l'argent à Guillaume Cade à Londres (4).

On peut du reste mesurer l'intensité des rapports commerciaux entre Saint-Omer et l'Angleterre à leurs relations religieuses et sociales.

Ce furent des marchands de Saint-Omer qui voyageaient en Angleterre tout autant que les moines de Saint-Bertin, qui répandirent en Flandre le culte de Saint Thomas Becket après 1170. Dans tout le comté on peut constater. une grande ferveur pour le nouveau saint : nulle part, on ne la remarque aussi bien qu'à SaintOmer. Guillaume, châtelain de Saint-Omer, qui avait peut-être été chargé par l'abbé de Canterbury de recueillir tous les récits de miracles de Saint Thomas, écrit

(1) Sur Guillaume CADE, ef. Jenkinson William Cade a financier of the XII century E. I. R. XXVIII, pp. 209-27. J.-H. Round The debtors of William Cade ibidem pp. 522-27 et Ch.-H. Haskins William Cade ibidem pp. 730-32.

(2) On les retrouve en effet comme témoins à Saint-Omer en 1166. Grry, op. cil., p. 386, P. J., $\mathrm{n}^{\circ} \mathrm{X} 111$.

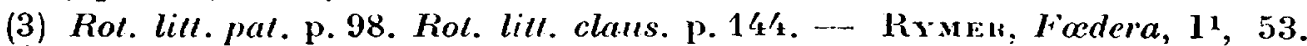
Sur Simon Saphir, il y a un excellent article de V. Fris dans la Biographie Nationale, t. XXI, pp. 384-87.

(1) Ce sont Willelmus Coterel, I,ambinus et Alardus de Oices, Malgerus Buc, Jerveus Farewcl, Willclmus Casc. Debita Willelmi Cade, éd. Jenlsinson, E. H. R., pp. 224-25. 
comment un voleur fut sauvé par celui-ci, près de SaintOmer (1). C'est en Angleterre sans doute que Saint Lambert, gros bourgeois de Saint-Omer apprit à invoquer Thomas qui, lors d'un grand festin, guérit un de ses invités (2). C'est à Canterbury même que Hugues, marchand de Saint-Omer fut guéri de la lèpre (3).

Entre Saint-Omer et l'Angleterre, c'est un va-et-vient continuel. L'abbaye de Saint-Bertin y est pour beaucoup, car elle reçoit toujours généreusement les nobles et les ecclésiastiques étrangers. Jean de Salisbury, envoyé de Thomas Becket, passe par Saint-Omer en 1164 (4). Peu après, Herbert de Boseham va à Saint-Bertin pour y préparer la fuite de l'archevêque (5). Lorsqu'il a réussi à s'évader de l'Angleterre, Becket lui-même vient passer quelques jours à Saint-Omer et à Saint-Bertin : il y reçoit, la même cordiale hospitalité qu'Anselme en 1097 (6). C'est à Saint-(Omer que le fameux chroniqueur Gérard de Barri dépose de l'argent en prévision de son voyage à Rome (7) ; il y passe à plusieurs reprises, y note la présence de nombreux anglais (8) et remarque que Hubert Walter, archevêque de Canterbury, exerce une véritable influence à Saint-Omer et dans tous les ports de Flandre (9).

C'est dans cette ville de Saint-Omer, remplie de bour-

(1) Guillaume de Ca vternury, op.cit., éd. Robertson Materials, I, p. 515.

(2) Benedictus, Miracula $S$. Thomae, éd. Robertson materials, II, p. 197.

(3) Guillaume de Cavtranury, op. cit., éd. Robertson, I, p. 332.

(4) Epistolae S. Thomae, no 55, ed. Robertson, V. pp. 96-97.

(5) Ilerbert de Bosenam, Vita S. Thomae, éd. Robertson, 111 , p. 313.

(6) Guillaume de Cavmenbury, op. cit., éd. Robertson, I, p. 42. - Cirim,

Vila S. Thomae, éd. Robertson, II, p. 400. Pour Anselme, cf. note 3, p. 316.

(7) Giraldus Cambrensis, Op. ommia, éd. Brewer. R. S. III, p. 239.

(8) Ibidem, ᄂ. I, pp. 50, 81, 118 et t. III, pp. 240-11.

(9) «Cantuariensis ccclesiac... potentiam quae in partibus illis et portubus Flandriae maritimis non nodica fuit...".

Ibidem, t. II I, p. 239. Sur l'influence et le rôle d'ITubert Warter, cf. Davis, England under the Normans and Angevins, p. 321 sqq. et p. 3/7. Ajoutons que Saint Bertin eut toujours d'étroites relations avec Caxtenuuny. Epistolae Canfuarienses, éd. STubus, memorials for the reign of Richard, I, t. II, pp. 156, 187, 260, 272, 415. 
LES MARCHAN DS FLAMANDS ETLE ROI D'ANGLETERRE 319

geois qui s'adonnent au commerce d'outre-Manche (1) que vécut Florent-le-Riche.

Sa famille est une de celles qui se sont le plus enrichies en Angleterre. Lui-même, par ses fructueux voyages dans l'île, par ses opérations commerciales à Saint-Omer même, par ses prêts au roi d'Angleterre, va la rendre plus influente encore. Au milieu du XIIIe siècle, beaucoup de ses membres feront partie de la hanse et occuperont des charges importantes dans la ville (2).

Nous ignorons tout des débuts de sa vie active, et les textes qui le concernent datent surtout du commencement du XIIIe siècle, époque à laquelle il devait être déjà assez âgé. Mais il est hors de doute que dès la fín du XIIe siècle, il était en rapports fréquents avec l'Angleterre: le fait qu'en 1197-98, Richard Ier lui faisait rembourser 500 livres d'Anjou qu'il lui avait empruntées prouve suffisamment que notre marchand était bien connu du roi et qu'il était, dès cette époque, un habitué des foires anglaises (3). Bientôt après d'ailleurs, nous pouvons le suivre dans ses différents voyages dans l'île.

Le 18 mai 1200 , Jean sans Terre avait fait savoir aux Flamands qu'ils pouvaient aller en touta sécurité en Angleterre ( 4 ). Florent ne se contente pas de cette autorisation importante sans doute, mais qui ne donne droit à aucune protection spéciale. d'autant plus nécessaire que la guerre menace d'arrêter tout commerce. Aussi profite-t-il du passage du roi Jean à Pont-de-l'Arche (11 mai 1202, Normandie) pour lui demander une lettre de protection particulière. A force d'argent, sans doute - comme c'était l'usage en pareil cas - il obtient une

(1) Liste presque complète dans Grry op. cit., p. 316 sqq.

(2) A. IIermand et L. Deschamps de Pas, Histoire sigillaire de SaintOmer, Paris, 1841, in-4. p. 42.

(3) "Et Florentio de Sancto Audomaro cct XXV li pro D. li. Andegas. quas Commodavit. regi". Public Record office. Exch. L. T. R. Pip. Roll. 40 Rot. 12 membr. 2 .

(1) Rot. litt. pat., p. 60 b et 64 . 
licence personnelle de commercer, valable pour un an "sive sit pax sive guerra"(1).

Puis, jusqu'en 1206, il ne demande plus rien au roi : le 6 juin 1204, Jean sans Terre avait de nouveau accordé une licence générale de commercer —à condition de payer le nouvel impôt du quinzième (2). Florent s'en contente. Mais en mai 1206, il achète mille marcs-poids de cuirs et de laine à l'évêque de Norwich. Comme il est impossible d'exporter de la laine sans permission spécịale, il est bien forcé de recourir au roi. Pour en finir plus vite - et à meilleur compte peut-être -.. il demande à l'évêque de Norwich d'intervenir auprès de Jean sans Terre : le 26 mai, celui-ci lui permet d'emporter sa laine en Flandre (3).

Nous le retrouvons en Angleterre dès 1207. Cette fois, il s'est adjoint un associé, Henri de Boulogne, marchand de Saint-Omer, en attendant que son fils Guillaume puisse voyager avec lui : le 14 juin, tous deux reçoivent des lettres de protection d'autant plus importantes qu'elles sont valables jusqu'à nouvel ordre et non plus pour un an comme en 1202 (4). Est-ce une preuve que Florent est décidément un favori du roi Jean? On pourrait le croire, car en 1207 celui-ci le charge de lui procurer trois on quatre chevaux par l'intermédiaire de son émissaire habituel, Walter de Baillolet (5).

Nous ignorons si Florent a pris une part très grande à l'action des villes en 1208 . On peut le supposer puisqu'en 1209, nous le trouvons en excellents termes avec le roi qui lui emprunte 100 mares d'argent pour un achat de chevaux (6).

(1) Rot. litt. pat., p. 10 b.

(2) Rot. litt. pat., p. 43.

(3) Rot. litt. pat., p. 65 b.

(1) Rot. litt. pat., p. 73.

(5) Rot. litl. pat., p. 77.

(6) Rot. litl. pat., p. $90 \mathrm{~b}$. 
Mais la situation politique ne cesse de s'embrouiller et en 1212, au moment où Ferrand de Portugal devient comte de Flandre, Louis (VIII) s'empare d'Aire et de Saint-Omer; en mars 1212, les échevins et la commune de Saint-Omer lui jurent fidélité (1). Du coup, la ville n'a plus rien à espérer du roi Jean; Florent moins que les autres, car il est probablement échevin et comme tel a joué un rôle dans la réception du prince Louis (2).

lmpossible dans ces conditions de songer à aller en Angleterre: Florent cherche une compensation et se fait donner en fief par le prince Louis l'ancienne gildehalle, les étaux des bouchers et deux maisons que Baudouin IX lui avait déjà concédées (3).

Puis nous le perdons de vue (4). La situation est décidément trop mauvaise pour qu'on le revoie dans l'île. Quand on retrouve sa trace, Guillaume le Maréchal, régent d'Angleterre, a fait avec lui un accord pour le remboursement des 6.000 mares que Henri II devait au prince Louis (5). Fut-ce une bonne affaire pour lui ? On peut en douter, car le remboursement total n'était pas effectué en 1224 (6). Pourtant, Florent trouva dans cette opération financière un grand avantage : celui de

(1) Deriste, Catalogue des actes de Philippe Auguste, p. 308, No 1359.

(2) Il était échevin en 1210, Haigneré, Chartes de Saint-Bertin, I, p. 219, No $^{\circ} 502$.

(3) L. Deschamps, Essai histor. sur l'hôtel de ville de Saint-Omer. Mém. Soc. Antiq. Mor., IV, p. 354, P. J., No 7, et Grry op. cit. p. 287.

(4) Giry qui consacre quelques lignes à Florent (Hist. de Saint-Omer, p. 316) ne semble pas avoir lu les documents avec attention. C'est ainsi qu'il cite un texte du 8 avril 1202 (Rot. lill. pat., p. 9) : il y est question de Johanni filio Ricardi de Sancto Homero et non de Florent comme il le croit. De même il se sert d'un texte de 1212 (Rot. litt. claus., p. 119), qui concerne, d'après lui, Guillaume, fils de Florent : or il s'agit sans aucun doute de Guillaume, châtelain de Saint-Omer.

(5) Patent Rolls of Henry $I I I$, t. I, p. 115. Bien qu'aucun traité ne parle d'une indemnité à accorder au prince Louis par Henri III, il est certain que celui-ci promit 10.000 marcs à son adversaire. 4.000 mares furent payés avant le départ du prince. Pexir-Dutaillis, Louis VIII, pp. 176-177.

(6) Au 31 mai 1224, le roi paie encore $38 \mathrm{~m} .3$ s. 4 d. à Guillaumc, fils de Florent. Rot. liit. claus, t. I, p. 602. 
pouvoir aller souvent en Angleterre, d'y acheter tout ce qu'il voulait et d'y obtenir facilement des marchandises. C'est ainsi qu'en septembre 121.7, le roi lui vendait 100 lests (lastos) de cuir et 100 sacs de laine pour payer une partie de sa dette (1). Filorent recevait en même temps un sauf-conduit pour lui, pour son fils et pour six bourgeois de Saint-Omer (2). Le 2 avril 1218 il en avait un nouveau, cette fois pour lui seul (3) : ce fut le dernier voyage qu'il fit en Angleterre. Son fils qui l'avait rejoint dans l'île en mai 1218 (4) termine la liquidation de la dette royale : car Florent mourut vraisemblablement avant le 29 aôt $1218(5)$.

Telle est la vie commerciale de ce grand marchand flamand du début du XIIIe siècle : elle est bien caractéristique de la vie économique, lelle qu'elle a été résumée plus haut.

Si l'histoire des relations économiques anglo-flamandes à la fin du XIIe et au début du XIIIe siècle est d'un si grand intérêt, c'est qu'alors le commerce entre la Flandre et l'Angleterre présente pour la première fois les caractères qu'il gardera pendant plusieurs siècles.

Sous Henri ler, les villes anglaises n'étaient que peu influentes, le roi se gardait de surveiller les marchands étrangers et se contentait, pour s'attirer la sympathie du comte de Flandre, de lui donner un fief de bourse.

(1) Le 21 septembre 1217. Patent Rolls of Henry III, t. I, P. 11'.

(2) Patent Rolls of Henry III, t. I, p. 114.

(3) Ibidem, p. 146.

(4) Le 16 mai, Henri III lui donne 40 sous "ad expensas suas", Rot. litt. claus., t. I, p. 362 .

(5) C'est probablement pour cela qu'à cette date le roi donne uno nouvelle reconnaissance de sa dette avec un étal des sommes qu'jl a déja payées. Patent Rolls of H enry III, t. I, p. 168. Pour pouvoir payer cette lourde dette, Menri III eut recours à de nombreux petits emprunts, à des saisies et à de nouvelles taxes, ef. Patent Rolls of Henry 111, p. 115. - - Rot. lill. claus, t. I, pp. $360 \quad b, 369,369 \quad b$ (ter), $381 \quad b, 383$ (bis), 388, 602. PerrtDutalllis, Louis VIII, p. 513. P. J. n. II e et p. 177. 
LES MAICHANDS FLAMANDS ETIEROI D'ANGLETERRE 323

Entre 1154 et 1213 au contraire, le monopole commercial des villes anglaises s'établit définitivement, le roi d'Angleterre organise le contrôle du commerce étranger et intervient grâce à une pression économique, dans la politique extérieure du comté voisin ; dès cette époque aussi, la laine est devenue la base essentielle du commerce anglo-flamand. II y aura bien encore des changements dans les relations économiques des deux pays : ainsi vers la fin du XIIIe siècle, les marchands de Flandre laisseront aller leur flotte à la ruine, s'adonneront uniquement au commerce passif et se feront apporter chez eux la laine anglajse; en outre, l'intensité des rapports commerciaux au début du XIIIe siècle est loin d'être comparable -.-pour autant que les sources nous permettent de le constater - à celle du XIVe siècle.

Mais il n'en reste pas moins vrai que dès le début du XIIIe siècle, les relations commerciales entre la grande île et le comté, ont l'aspect général qu'elles conserveront pendant longtemps. Il reste surtout que la politique de pression économique inaugurée par Henri II sera suivie par tous ses successeurs et aura une importance capitale pour l'histoire politique du XIVe siècle.

Vers 1200 , les marchands flamands pour sauvegarder leurs intérêts ont dû se plier devant Jean sans Terre; en tant que corps, ils ont eu une politique manifestement favorable à ce roi ; en tant qu'individus, ils se sont efforcés de flatter ses manies, de combler ses désirs, de lui complaire en toutes choses. Au XIVe siècle, les tisserands et les foulons gantois, conduits par Jacques Van Artevelde, n'agiront pas autrement.

Evidemment, à l'enjeu on peut mesurcr lc chemin parcouru et juger de l'extension qu'a pris le commerce de la laine : en 1208, les gros marchands flamands n'ont à sauver que leur fortune, au XIVe siècle, tout un prolé- 
tariat urbain veut, en se soumettant au roi d'Angleterre, éviter la famine et la mort.

Mais on peut dire à juste titre que le commerce angloflamand du XIVe siècle n'est que le développement rationnel de celui du XIIIe.

Gaston Dept. 